\title{
Purification of polysaccharides from Cordyceps militaris and their anti-hypoxic effect
}

\author{
YUAN DONG ${ }^{1,2}$, SHUANG HU$^{1}$, CHUNGANG LIU $^{1}$, QINGFAN MENG ${ }^{1}$, JIA SONG $^{1}$, JIAHUI LU $^{1}$, \\ YINGKUN CHENG ${ }^{1}$, CHAOHUI GAO $^{1}$, YAN LIU ${ }^{1}$, DI WANG ${ }^{1}$ and LIRONG TENG ${ }^{1,3}$ \\ ${ }^{1}$ College of Life Science, Jilin University, Changchun, Jilin 130012; ${ }^{2}$ Academy of Laboratory, Jilin Medical College, \\ Jilin, Jilin 132013; ${ }^{3}$ College of Life Science, Zhuhai College, Jilin University, Zhuhai, Guangdong 519000, P.R. China
}

Received January 20, 2014; Accepted September 4, 2014

DOI: $10.3892 / \mathrm{mmr} .2014 .2786$

\begin{abstract}
Acute mountain sickness, one of the most common altitude diseases, causes lung and brain injury. The present study aimed to investigate the anti-hypoxic effect of purified polysaccharides extracted from Cordyceps militaris. The aqueous extract of Cordyceps militaris was purified progressively through a DEAE-52 cellulose anion exchange column and a Sepharose G-100 column. The fraction CMN1, with a molecular weight of $37842 \mathrm{Da}$, was the main fraction obtained and its chemical composition and structural characteristics were determined. CMN1 was found to have a monosaccharide composition of L-rhamnose, L-arabinose, D-mannose, D-galactose. The backbone of CMN1 comprised $(1 \rightarrow 2)$ and $(1 \rightarrow 3)$ linkages, with branched $(1 \rightarrow 6)$ and $(1 \rightarrow 4)$ linkages. The anti-hypoxic effects of CMN1 were determined using a sodium nitrite toxicosis test, acute cerebral ischemic/hypoxic test and normobarie hypoxia test. CMN1 $(0.5 \mathrm{~g} / \mathrm{kg})$ possessed a similar anti-hypoxic effect to rhodiola oral liquid. Overall, the Cordyceps militaris polysaccharide, CMN1, was identified as an effective agent against hypoxia.
\end{abstract}

\section{Introduction}

As one of the most common altitude illnesses, acute mountain sickness (AMS) causes lung and brain injury, with symptoms including headache, loss of appetite, dizziness and insomnia (1). The decreased barometric pressure and subsequent reduction in available oxygen are the primary causal factors of AMS (2). Long-term hypoxia causes irreversible damage and ultimately

Correspondence to: Dr Di Wang, College of Life Science, Jilin University, 2699 Qianjin Street, Changchun, Jilin 130012, P.R. China E-mail: jluwangdi@gmail.com

Professor Lirong Teng, College of Life Science, Zhuhai College, Jilin University, 286 Jinhai Road, Zhuhai, Guangdong 519000, P.R. China

E-mail: tenglr@jlu.edu.cn

Key words: Cordyceps militaris, polysaccharides, purification, structural analysis, anti-hypoxic effect leads to organ failure. Currently, the normal vasodilating agents, nifedipine and acetazolamide, are used as prophylactic agents to reduce the incidence and severity of AMS at high altitudes (3). However, serious adverse effects, including headache and cardiopalmus have been observed during the clinical treatment (4), creating a demand for the identification of alternative agents.

Cordyceps militaris, which belongs to the class Ascomycetes and the Dong Chong Xia Cao group of Chinese herbs, possesses potential antioxidant (5), immunomodulatory (6), antitumor and anti-inflammatory properties (7). In Western countries, Cordyceps militaris is considered to be a Chinese herb with anti-aging and anticancer effects (8). Several studies have investigated the polysaccharides of Cordyceps, which are a rich and important activity group. One Cordyceps militaris polysaccharide-enriched fraction produced hypoglycemic activity (9) and reduced plasma glucose in normal Wistar-Kyoto rats (10). In our previous study, Cordyceps militaris polysaccharides exhibited significant anti-diabetic and anti-nephropathic activities (11). As reported previously, the bioactivities of polysaccharides are associated with their chemical composition, glycosidic linkages, conformation, molecular weight and degree of branching (12). Previous studies have examined different polysaccharides extracted from the Cordyceps militaris sporocarp and these are comprised mainly of mannose, rhamnose, galactose and glucose and have an average molecular weight of $30 \mathrm{kDa}$ (13-15). However, few studies have investigated the purification and bioactivities of polysaccharides separated from Cordyceps militaris mycelium, which is cultured by submerged fermentation $(16,17)$. Submerged fermentation presents the advantage of simultaneously enabling a decrease in disposal costs and the production of value-added products and, thus, has been widely used.

In the present study, polysaccharides extracted from Cordyceps militaris mycelium were purified and characterized. Furthermore, the anti-hypoxic effects of the purified Cordyceps polysaccharides were detected in vivo.

\section{Materials and methods}

Strain and reagents. The study was approved by the ethics committee of the Lab Animal Center of Jilin University; ref no. 2009-0011). Cordyceps militaris was purchased from the National Biological Resource Center (Chiba, Japan; cat 
no. NBRC9787). Sodium nitrite, glucose, peptone, yeast extract powder, $\mathrm{H}_{2} \mathrm{SO}_{4}, \mathrm{NaIO}_{4}$, acetonitrile, glycol (analytical grade), $\mathrm{NaOH}$, potassium hydrogen phthalate, acetic acid, potassium borohydride, $\mathrm{BaCO}_{3}, \mathrm{KH}_{2} \mathrm{PO}_{4}, \mathrm{MgSO}_{4} .7 \mathrm{H}_{2} \mathrm{O},(\mathrm{NH} 4)_{2} \mathrm{SO}_{4}$, $\mathrm{ZnCl}_{2}$, Vitamin $\mathrm{B} 1$ and dextran standards were all obtained from Sigma-Aldrich (St. Louis, MO, USA).

Submerged incubation and fermentation. Cordyceps militaris was cultured in a rotary shaker incubator $(10 \mathrm{~L}$, Biostat B; Sartorius AG, Goettingen, Germany) at $150 \mathrm{rpm}$ for 5 days at $26^{\circ} \mathrm{C}$. The cultured medium contained $20 \mathrm{~g} / 1$ glucose, $10 \mathrm{~g} / 1$ peptone, $18 \mathrm{~g} / 1$ yeast extract powder, $3 \mathrm{~g} / 1 \mathrm{KH}_{2} \mathrm{PO}_{4}$, $3 \mathrm{~g} / 1 \mathrm{MgSO}_{4} 7 \mathrm{H}_{2} \mathrm{O}, 10 \mathrm{~g} / \mathrm{l}(\mathrm{NH} 4)_{2} \mathrm{SO}_{4}, 0.01 \mathrm{~g} / 1 \mathrm{ZnCl}_{2}$ and $0.24 \mathrm{~g} / 1$ vitamin $\mathrm{B} 1$. The mycelia were then harvested and lyophilized for further use.

Crude extract preparation and preliminary identification. As described previously (18), the aqueous extract from Cordyceps militaris was prepared by extracting $100 \mathrm{~g}$ mycelia powder twice using hot water at $80^{\circ} \mathrm{C}$ for $3 \mathrm{~h}$. Following centrifugation at $4,500 \times \mathrm{g}$ for $10 \mathrm{~min}$, the supernatant was sequentially concentrated and freeze-dried to produce the solid aqueous extract of Cordyceps militaris (CM (indicating the Cordyceps militaris extract)). According to the previously described method (19), different chromogenic reactions were used to determine the constituents of CM.

Purification of the CM. Using Sevag reagent [V (n-butanol): $\mathrm{V}$ (chloroform)=1:4, $50 \mathrm{ml}$, Sigma-Aldrich, St. Louis, MO, USA], the proteins present in the CM were removed (20). Ethanol (4-fold) was added to the supernatant, which was placed at $4^{\circ} \mathrm{C}$ overnight. The precipitation was then dissolved in double distilled (DD) water and placed in a $2.6 \mathrm{~cm} \times 35 \mathrm{~cm}$ DEAE-52 cellulose anion exchange column (21). The column was eluted with DD water, followed by 0.1 and $0.3 \mathrm{~mol} / 1 \mathrm{NaCl}$, respectively, at a flow rate of $1 \mathrm{ml} / \mathrm{min}$. The polysaccharide fraction was collected and detected using an anthrone acid method (19). The gel permeation chromatography system, Sepharose G-100 (General Electric Co.Salt Lake City, Utah, USA) was used for further purification. The column was eluted with DD water at a flow rate of $0.4 \mathrm{ml} / \mathrm{min}$. The fractions (10 ml each) were then collected (13) and freeze-dried.

Fourier transform infrared spectroscopy (FTIR) determination. The purified polysaccharides (4 mg) were ground thoroughly using $150 \mathrm{mg} \mathrm{KBr}$ (Sigma-Aldrich). The average transmission spectra $(n=50)$ were recorded using an IRPrestige-21 FTIR spectrometer (Shimadzu Corporation, Tokyo, Japan) between 400 and $4,000 \mathrm{~cm}^{-1}$ and the absorbance was determined.

Homogeneity and molecular weight determination. The homogeneity and molecular weight were analyzed using a high performance liquid chromatography (HPLC)/evaporative light scattering detector (ELSD) system (22). An LC-10ATvp HPLC system (Shimadzu Corporation) equipped with a TSKgel G4000PWXL column (Tosoh, Tokyo, Japan) and an Alltech 2000ES ELSD (Shimadzu Corporation) was used. Briefly, DD water served as the mobile phase, which was driven by a double pump (Waters 150; Millipore, Billerica, MA, USA) at a flow rate of $0.45 \mathrm{ml} / \mathrm{min}$. The aerosol level was
$60 \%$, the drift tube temperature was $120^{\circ} \mathrm{C}$ and the nebulizing nitrogen pressure was 25 psi. Dextran standards were used to create a calibration curve, as previously described (23).

Monosaccharides analysis. The analysis of monosaccharides was investigated according to the previous method (24). The polysaccharide $(20 \mathrm{mg})$ was hydrolyzed with $1 \mathrm{M} \mathrm{H}_{2} \mathrm{SO}_{4}$ $(1 \mathrm{ml})$ for $6 \mathrm{~h}$ at $105^{\circ} \mathrm{C}$ in a sealed glass tube and the $\mathrm{pH}$ was adjusted to 7.0 using $\mathrm{BaCO}_{3}$. The solution was then centrifuged at 3,200 x g for $10 \mathrm{~min}$ to separate the hydrolysates, which were further analyzed using the HPLC/ELSD system. The chromatograph was fitted with a Prevail ${ }^{\mathrm{TM}}$ ES carbohydrate analysis column (Alltech Associates, Inc., Deerfield, IL, USA), which was eluted with $75 \%$ acetonitrile (Sigma-Aldrich) at a flow rate of $1.0 \mathrm{ml} / \mathrm{min}$. The results were compared with the following monosaccharide standards: D-glucose, L-rhamnose (Rha), D-xylose, D-galactose (Gal), D-mannose (Man) and L-arabinose (Ara), trehalose (Sigma-Aldrich) (25).

Periodate oxidation-Smith degradation reaction of polysaccharides. The polysaccharide (20 $\mathrm{mg}$ ) was dissolved in $15 \mathrm{mM}$ $\mathrm{NaIO}_{4}\left(25 \mathrm{ml}\right.$; pH 4) in darkness at $4^{\circ} \mathrm{C}$. Subsequently, $100 \mu \mathrm{l}$ was withdrawn at $6 \mathrm{~h}$ intervals, diluted with distilled water and measured spectrophotometrically at $223 \mathrm{~nm}$ until a stable absorbance was reached (26). The consumption of $\mathrm{HIO}_{4}$ was calculated and the production of formic acid was determined by titration with $0.005 \mathrm{M} \mathrm{NaOH}$ and glycol $(2 \mathrm{ml})$ was added to terminate the oxidation reaction. The remaining periodate product was then fully dialyzed against DD water for $48 \mathrm{~h}$. This dialysate was concentrated and reduced with potassium borohydride (70 mg) overnight at room temperature and the $\mathrm{pH}$ was adjusted using acetic acid (Sigma-Aldrich) to $\mathrm{pH}$ 7.0. The solution was dialyzed against DD water for $24 \mathrm{~h}$ and $3 \mathrm{ml}$ sample was further analyzed using the HPLC/ELSD system. The remaining product was hydrolyzed using $1 \mathrm{M} \mathrm{H}_{2} \mathrm{SO}_{4}$ at $25^{\circ} \mathrm{C}$ for $40 \mathrm{~h}$ and was adjusted to $\mathrm{pH} 7.0$ using $\mathrm{BaCO}_{3}$. Following centrifugation at 3,200 x g for $10 \mathrm{~min}$, the hydrolysates were analyzed using HPLC/ELSD under the same conditions that were used for the monosaccharide composition analysis.

In vivo experiments in an animal model of hypoxia. The experimental animal procedure was approved by the Lab Animal Centre of Jilin University [Changchun, China; License no. SCXK-(JI) 2006-0001)]. Mice weighing 18-22 g were maintained under a constant $12 \mathrm{~h}$ light/dark cycle at $23 \pm 1^{\circ} \mathrm{C}$, with a humidity of $60 \pm 2 \%$ with water and food available ad libitum. All mice were fed with standard laboratory feed in an animal room for 3 days prior to the experiments. To produce experimental models of hypoxia, 180 mice (90 males and 90 females) were randomly divided into three groups for the normobarie hypoxia test, sodium nitrite toxicosis test and acute cerebral ischemic/hypoxic test (27), respectively. The normobarie hypoxia test was performed in a sealed wide-mouth bottle containing soda lime (Sigma-Aldrich). The sodium nitrite toxicosis test was performed by injection of $2 \mathrm{~g} / \mathrm{ml}$ sodium nitrite. The acute cerebral ischemic-hypoxic test was performed by decapitation of the mice. In all three tests, mice received either physiological saline or $1.5 \mathrm{ml} / \mathrm{kg} / \mathrm{day}$ rhodiola oral liquid, which served as the normal control (NC) group or the positive 
control (PC) group. The remaining mice were divided into three groups $(\mathrm{n}=12)$ and treated orally with $\mathrm{CMN} 1(0.0 \mathrm{~g} / \mathrm{kg}$, $0.2 \mathrm{~g} / \mathrm{kg}$ or $0.5 \mathrm{~g} / \mathrm{kg}$ ) once a day. After 24 days, the survival rate of hypoxia under normal pressure and following sodium nitrite injection were determined and the persistence of gasping following decapitation was examined.

Statistical analysis. All data are expressed as the mean \pm standard deviation. Data were evaluated using one-way analysis of variance to detect statistical significance, followed by post-hoc multiple comparisons (Dunn's test). $\mathrm{P} \leq 0.05$ was considered to indicate a statistically significant difference.

\section{Results}

Phytochemical assessment. The constituents of the Cordyceps militaris extract, including proteins, organic acid and sugars were detected (Table I). At room temperature, organic acid was unstable, which suggested that the major effective components in the Cordyceps militaris extract were protein and polysaccharides. In addition, the content of the total polysaccharides was $163 \mathrm{mg} / \mathrm{g}$ in the cultured Cordyceps militaris mycelium.

Purification and characterization of polysaccharides. The neutral or acidic polysaccharides in Cordyceps militaris water extracts were separated using anion exchange chromatography in a DEAE-cellulose column. Two fractions, CM1 and CM2, were eluted using DD water and $0.1 \mathrm{M} \mathrm{NaCl}$, respectively and the yields of CM1 and CM2 were 0.475 and $0.025 \mathrm{~g} / \mathrm{g}$, respectively (Fig. 1A). When examining the rate of production, only CM1 was further purified using the gel permeation chromatography system Sepharose G-100. A single elution peak (CMN1) appeared at $60 \mathrm{~min}$ (Fig. 1B) with a molecular mass of 37,842 Da (Fig. 2A and B). Rha, Ara, Man and Gal were present in CMN1, according to the retention time in the HPLC figure print (Fig. 2C and D). The molar ratio of Rha, Ara, Man and $\mathrm{Gal}$ was 1.48:11.34:11.62:1.00.

Furthermore, the characteristic structure of CMN1 was analyzed using FTIR and periodate oxidation-Smith degradation. In the FTIR spectra (Fig. 3), peaks at 3,494, 2,900, 1,627, $1,558,1,458,1,006$ and $524 \mathrm{~cm}^{-1}$ were noted.

Periodate oxidation-Smith degradation was performed to confirm the linkage mode of the glucose present in CMN1 (Fig. 4) and the HPLC method was performed to analyze the products following Smith degradation. Rha and Ara were noted prior to hydrolysis, however, Ara, glycerol and erythritol were observed following hydrolysis (Fig. 4B).

Anti-hypoxic effects of CMN1. In the normobarie hypoxia test and acute cerebral ischemic-hypoxic test, mice were sacrificed by suffocation. In sodium nitrite-induced acute acquired methemoglobinemia, less oxygen is carried by hemoglobin, which leads to tissue death (25). Compared with the NC group, $0.5 \mathrm{~g} / \mathrm{kg}$ CMN1 improved survival rate by almost 53\% $(\mathrm{P}<0.05)$ in the sodium nitrite intoxication survival test and by $39 \%$ in the hypoxia-resistance test $(\mathrm{P}<0.05)$, respectively (Fig. 5A and C). Furthermore, treatment with $0.5 \mathrm{~g} / \mathrm{kg}$ CMN1 resulted in a 1.88 -fold increase in gasping persistence $(\mathrm{P}<0.01$; Fig. $5 \mathrm{~B})$. No significant
Table I. Summary of the preliminary chemical analysis of crude extracts.

\begin{tabular}{lr} 
Test & CM \\
\hline Amino acid/polypeptide/protein & + \\
Soluble reducing sugar & + \\
Sugars & + \\
Phenolics/tannin & - \\
Alkaloids & - \\
Sterols & - \\
Terpenes & - \\
Organic acid & + \\
Essential oil/oil & - \\
Anthraquinone & - \\
Flavonoids & - \\
Coumarin/lactone & -
\end{tabular}

'+' detected; '-' undetected; CM, Cordyceps militaris extract.

differences were observed between the CMN1-treated and PC groups indicating that CMN1 possesses an anti-hypoxic effect similar to that of rhodiola oral liquid.

\section{Discussion}

AMS, which produces a large number of oxygen radicals, leads to irreversible tissue damage (29). In previous clinical trials, Sipunculus nudus L. (27), Brassica rapa L. (28), Rhodobryum giganteum Par. (30) and rhodiola were used to alleviate the symptoms of AMS. In the present study, Cordyceps polysaccharides were purified and characterized and their anti-hypoxic effects were detected in vivo.

In the FTIR spectra, a broad stretching peak was observed at $\sim 3,494 \mathrm{~cm}^{-1}$ and a weak peak at $\sim 2,900 \mathrm{~cm}^{-1}$. These are characteristic absorption peaks of saccharides and were produced by marked hydroxyl group vibration (31) and by $\mathrm{C}-\mathrm{H}$ bending vibration of the $-\mathrm{CH}_{2}$ groups (32), respectively. The bands at 1,627 and $1,558 \mathrm{~cm}^{-1}$ suggested the existence of a $\mathrm{C}=\mathrm{O}$ bond and an $\mathrm{N}-\mathrm{H}$ bond, possibly from the amidogen in Gal. The weak peak at $1,458 \mathrm{~cm}^{-1}$ was attributed to vibrations of $\mathrm{CH}_{3}$ and an absorption peak at 1,006 and $524 \mathrm{~cm}^{-1}$, may have been contributed to by the ether linkage (C-O-C) and the hydroxyl in the pyranose ring.

Analysis of the results obtained in the periodate oxidation-Smith degradation suggested the possible linkage of monosaccharides within CMN1. The consumption of $<1 \mathrm{~mol}$ periodate $(0.82 \mathrm{~mol})$ in the present study indicated the existence of a $1 \rightarrow 3$ linkage, which does not consume periodate during oxidation. Following periodate oxidation, the presence of Rha and Ala revealed that CMN1 contained a section of $(1 \rightarrow 3)$-linked-Rha and $(1 \rightarrow 3)$-linked-Ala. The monosaccharide ratio suggested that the majority of $(1 \rightarrow 3)$-linked-Ala is located in the backbone and the $(1 \rightarrow 3)$-linked-Rha may be located in the backbone or the side chains. Formic acid production during periodate oxidation confirmed the existence of a $1 \rightarrow 6$ linkage. The low formic 

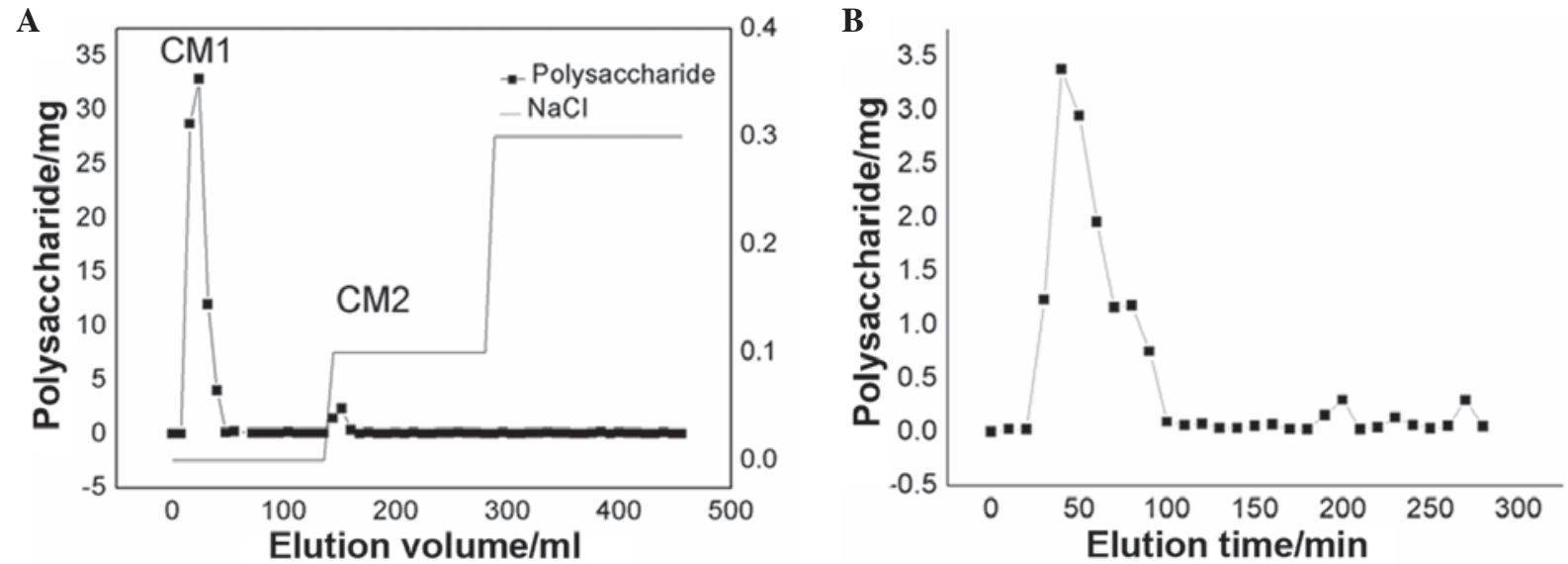

Figure 1. (A) DEAE-Sepharose fast flow chromatogram of the crude polysaccharides. CM1 was eluted using DD water and CM2 was eluted using $0.1 \mathrm{M} \mathrm{NaCl}$ at a flow rate of $1.0 \mathrm{ml} / \mathrm{min}$. CM1 $(5.0 \mathrm{ml})$ and CM2 $(5.0 \mathrm{ml})$ were obtained using a fraction collector. (B) CM1 was purified using Sepharose G-100. The column was eluted using DD water at a flow rate of $0.4 \mathrm{ml} / \mathrm{min}$. DD, double distilled; CM, Cordyceps militaris extract.

$\mathbf{A}$

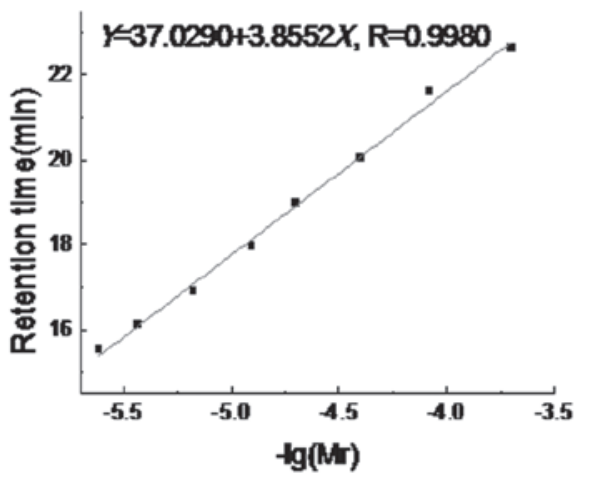

C

\begin{tabular}{cc}
\hline Monosaccharide & $\begin{array}{c}\text { Retention time } \\
(\mathrm{min})\end{array}$ \\
\hline L-rhamnose & 6.932 \\
L-arabinose & 7.523 \\
D-xylose & 8.281 \\
D-mannose & 8.928 \\
D-galactose & 12.444 \\
D-glucose, & 13.445 \\
trehalose & 28.735 \\
\hline
\end{tabular}

B

D
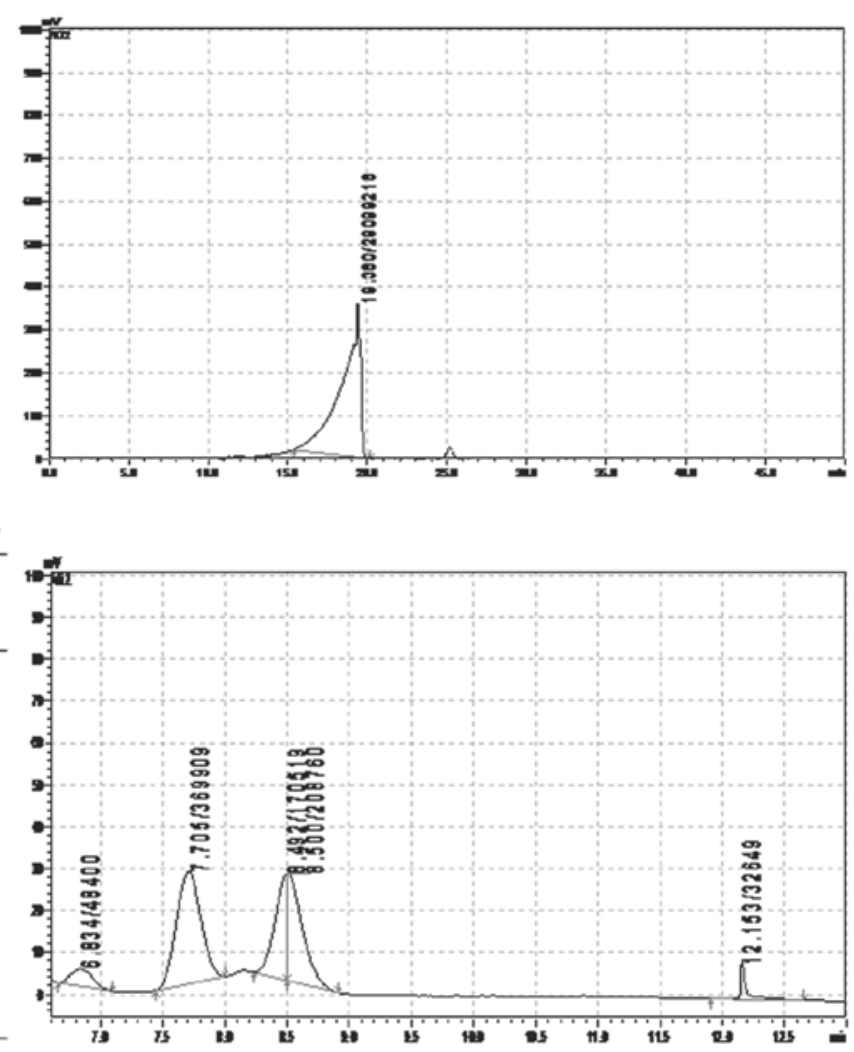

Figure 2. (A) Calibration curve of standard dextrans for CMN1 molecular weight determination. (B) Molecular weight of CMN1 was determined using a HPLC/ELSD system equipped with a TSK-GEL G4000PWXL column. (C) Retention time of the standard monosaccharide mixture (Rha; Xyl; Ara; Man; Glc; Gal). (D) Monosaccharide compositions of CMN1 were analyzed using an HPLC/ELSD system. HPLC, high performance liquid chromatography; ELSD, evaporative light scattering detector.

acid yield $(0.06 \mathrm{~mol} / \mathrm{sugar}$ residue, less than $0.1 \mathrm{~mol} / \mathrm{sugar}$ residue) suggested that the $1 \rightarrow 6$ linkage in CMN1 was unimportant. In addition, the $1 \rightarrow 2$ linkages may have been the main chain linkage of CMN1, which was indicated by the high-yield glycerol production. A trace quantity of erythritol revealed the low content of $1 \rightarrow 4$ linkages in the main chain or branch. Collectively, the backbone of CMN1 was found to be composed of $(1 \rightarrow 2)$ linkages and $(1 \rightarrow 3)$ linkages with branched $(1 \rightarrow 6)$ linkages and $(1 \rightarrow 4)$ linkages.
The normobarie hypoxia, sodium nitrite toxicosis and acute cerebral ischemic-hypoxic tests (24) were used to detect the anti-hypoxic activity of CMN1. Compared with the $\mathrm{NC}$ group, $\mathrm{CMN} 1$ markedly improved the survival rate in the sodium nitrite toxicosis and normobarie hypoxia tests. Enhanced gasping persistence was also observed in the acute cerebral ischemic-hypoxic models. Additionally, water extracts from Cordyceps sinensis have been found to exhibit a scavenging effect on reactive oxygen species, superoxide 


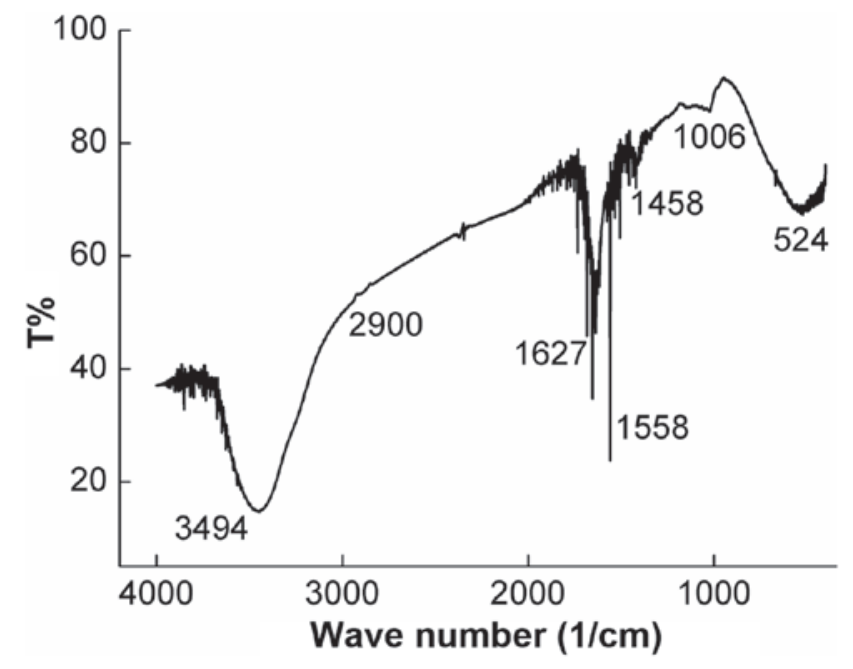

Figure 3. Fourier transform infrared spectroscopy spectrum of CMN1. T, transmittance.

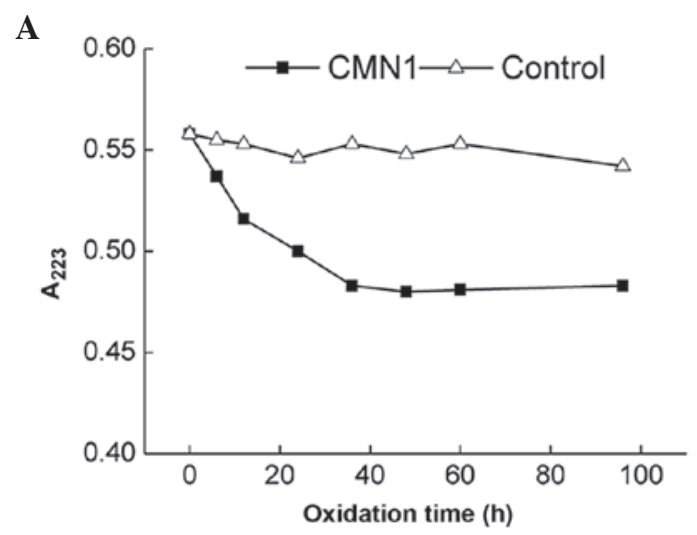

B

The HPLC analysis of Smith degradation products

\begin{tabular}{lcccccc}
\hline & Rha & Ara & Man & Gal & Glycerol & Erythritol \\
\hline Before hydrolysis & + & + & - & - & - & - \\
After hydrolysis & - & + & - & - & + & + \\
\hline
\end{tabular}

"+"Detected;"“-"Undetected

Figure 4. (A) Periodate oxidation time course. (B) Products of Smith degradation of CMN1 were detected using HPLC. HPLC, high performance liquid chromatography; Rha, L-rhamnose, Ara, L-arabinose; Man, D-mannose; Gal, D-galactose.

anions and hydroxyl radicals by inhibiting malondialdehyde formation (33). Overall, the polysaccharide extracted from Cordyceps militaris was also effective against hypoxia.

Furthermore, the anti-hypoxic activity of CMN1 may be associated with its characteristic structure. CMN1 was observed to have a molecular weight of 37,842 Da and its monosaccharide composition consisted of Rha, Ara, Man and Gal. Compared with previous studies, CMN1 exhibits a
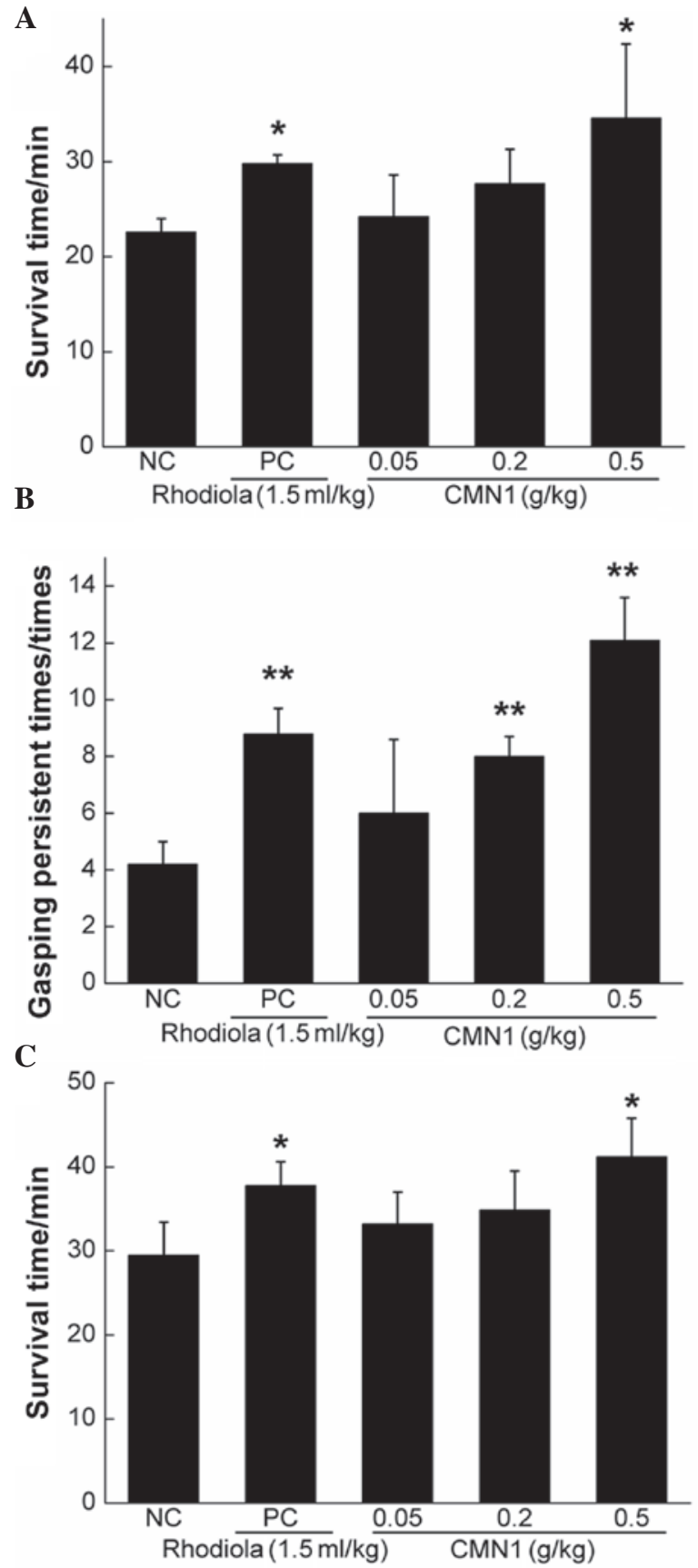

Figure 5. Anti-hypoxic effect of CMN1 determined using (A) sodium nitrite toxicosis, (B) acute cerebral ischemic-hypoxic and (C) normobarie hypoxia tests, respectively. The normobarie hypoxia test was performed in a sealed wide-mouth bottle containing soda lime. The sodium nitrite toxicosis test was performed by injection of $2 \mathrm{~g} / \mathrm{ml}$ sodium nitrite. The acute cerebral ischemic-hypoxic test was performed by decapitation of the mice. Data are expressed as the mean \pm standard deviation ( $n=10$ /group) and analyzed using one-way analysis of variance. ${ }^{*} \mathrm{P}<0.05$ and ${ }^{* *} \mathrm{P}<0.01$, vs. the $\mathrm{NC}$ group. NC, normal control; PC, positive control.

similar primary structure (14), however, additional studies are required to identify the pharmacological efficacy and characteristic structures of the polysaccharides.

The present study was limited to one polysaccharide-enriched fraction separated from Cordyceps militaris. Whether other components exhibit significant roles in hypoxia remains to be elucidated. Therefore, further investigations focusing on Cordyceps militaris are required. 


\section{Acknowledgements}

This study was supported by the twelfth Five-Year National key Technology R\&D Program (grant no. 2012BAI29B00).

\section{References}

1. Cueto-Martin B, De La Cruz-Marquez J and Garcia-Torres L: Effect of altitude in the blood pressure regulation system (renin-angiotensin-aldosterone) in team sports. Case study: Female volleyball. Med Sport 52: 261-269, 1999 (In Italian).

2. Fiore D, Hall S and Shoja P: Altitude illness: risk factors, prevention, presentation, and treatment. Am Fam Physician 82: $1103-1110,2010$

3. Wang J, Ke T, Zhang X, et al: Effects of acetazolamide on cognitive performance during high-altitude exposure. Neurotoxicol Teratol 35: 28-33, 2013.

4. Fagenholz PJ, Gutman JA, Murray AF and Harris NS: Treatment of high altitude pulmonary edema at $4240 \mathrm{~m}$ in Nepal. High Alt Med Biol 8: 139-146, 2007.

5. Li X, Mitchell J, Wood S, Coen C, Lightman S and O'Byrne K The effect of oestradiol and progesterone on hypoglycaemic stress-induced suppression of pulsatile luteinizing hormone release and on corticotropin-releasing hormone mRNA expression in the rat. J Neuroendocrinol 15: 468-476, 2003.

6. Kim CS, Lee SY, Cho SH, et al: Cordyceps militaris induces the IL-18 expression via its promoter activation for IFN-gamma production. J Ethnopharmacol 120: 366-371, 2008.

7. Rao YK, Fang SH, Wu WS and Tzeng YM: Constituents isolated from Cordyceps militaris suppress enhanced inflammatory mediator's production and human cancer cell proliferation. J Ethnopharmacol 131: 363-367, 2010

8. Paterson RR: Cordyceps: a traditional Chinese medicine and another fungal therapeutic biofactory? Phytochemistry 69 $1469-1495,2008$

9. Zhang G, Huang Y, Bian Y, Wong JH, Ng TB and Wang H: Hypoglycemic activity of the fungi Cordyceps militaris Cordyceps sinensis, Tricholoma mongolicum, and Omphalia lapidescens in streptozotocin-induced diabetic rats. Appl Microbiol Biotechnol 72: 1152-1156, 2006.

10. Cheng YW, Chen YI, Tzeng CY, et al: Extracts of Cordyceps militaris lower blood glucose via the stimulation of cholinergic activation and insulin secretion in normal rats. Phytother Res 26: 1173-1177, 2012.

11. Dong Y, Jing T, Meng Q, Liu C, Hu S, Ma Y, Liu Y, Lu J, Cheng Y, Wang D and Teng LR: Studies on the anti-diabetic activities of Cordyceps militaris extract in diet-streptozotocin-induced diabetic Sprague-Dawley rats. Biomed Res Int 2014: 160980, 2104 (Epub ahead of print)

12. Methacanon P, Madla S, Kirtikara K and Prasitsil M: Structural elucidation of bioactive fungi-derived polymers. Carbohydr Polym 60: 199-203, 2005.

13. Wu F, Yan H, Ma X, et al: Comparison of the structural characterization and biological activity of acidic polysaccharides from Cordyceps militaris cultured with different media. World J Microbiol Biotechnol 28: 2029-2038, 2012.

14. Yu RM, Yang W, Song LY, Yan CY, Zhang Z and Zhao Y: Structural characterization and antioxidant activity of a polysaccharide from the fruiting bodies of cultured Cordyceps militaris. Carbohydr Polym 70: 430-436, 2007.
15. Lee JS, Kwon JS, Won DP, et al: Study of macrophage activation and structural characteristics of purified polysaccharide from the fruiting body of Cordyceps militaris. J Microbiol Biotechnol 20: 1053-1060, 2010

16. Castilho LR, Mirchel DA and Freire DM: Production of polyhydroxyalkanoates (PHAs) from waste materials and by-products by submerged and solid-state fermentation. Bioresour Technol 100: 5996-6009, 2009.

17. Shih IL, Tsai KL and Hsieh C. Effects of culture conditions on the mycelial growth and bioactive metabolite production in submerged culture of Cordyceps militaris. Biochem Eng J 33: 193-201, 2007.

18. Du L, Song J, Wang H, et al: Optimization of the fermentation medium for Paecilomyces tenuipes N45 using statistical approach. Afr J Microbiol Res 6: 6130-6141, 2012.

19. Dhanabal S, Kokate C, Ramanathan M, Kumar E and Suresh B: Hypoglycaemic activity of Pterocarpus marsupium Roxb. Phytother Res 20: 4-8, 2006.

20. Yan H, Zhu D, Xu D, Wu J and Bian X: A study on Cordyceps militaris polysaccharide purification, composition and activity analysis. Afr J Biotechnol 7: 4004-4009, 2008.

21. Zhang Al, Lu JH, Zhang N, Zheng D, Zhang GR and Teng LR: Extraction, purification and anti-tumor activity of polysaccharide from mycelium of mutant Cordyceps militaris. Chem Res Chin Univ 26: 798-802, 2010 (In Chinese).

22. Zhang N, Liu Y, Lu J, et al: Isolation, purification and bioactivities of polysaccharides from Irpex lacteus. Chem Res Chin Univ 28: 249-254, 2012 (In Chinese).

23. Cui H, Chen Y, Wang S, Kai G and Fang Y: Isolation, partial characterisation and immunomodulatory activities of polysaccharide from Morchella esculenta. J Sci Food Agric 91: 2180-2185, 2011.

24. Chambers RE and Clamp JR: An assessment of methanolysis and other factors used in the analysis of carbohydrate-containing materials. Biochem J 125: 1009-1018, 1971.

25. Xie J, Xie M, Nie S, Shen M, Wang Y and Li C: Isolation, chemical composition and antioxidant activities of a water-soluble polysaccharide from Cyclocarya paliurus (Batal.) Iljinskaja. Food Chem 119: 1626-1632, 2010.

26. Linker A, Evans L and Impallomeni G: The structure of a polysaccharide from infectious strains of Burkholderia cepacia. Carbohydr Res 335: 45-54, 2001

27. Zhang CX and Dai ZR: Anti-hypoxia activity of a polysaccharide extracted from the Sipunculus nudus L. Int J Biol Macromol 49: 523-526, 2011.

28. Xie Y, Jiang SP, Su DH, Pi NN, Ma C and Gao P: Composition analysis and anti-hypoxia activity of polysaccharide from Brassica rapa L. Int J Biol Macromol 47: 528-533, 2010.

29. Hu D, Chen F, Guan C, Yang F and Qu Y: Anti-hypoxia effect of adenovirus-mediated expression of heat shock protein 70 (HSP70) on primary cultured neurons. J Neurosci Res 91: 1174-1182, 2013.

30. Cai Y, Lu Y, Chen R, Wei Q and Lu X: Anti-hypoxia activity and related components of Rhodobryum giganteum Par. Phytomedicine 18: 224-229, 2011.

31. Fang X, Jiang B and Wang X: Purification and partial characterization of an acidic polysaccharide with complement fixing ability from the stems of Avicennia marina. J Biochem Mol Biol 39: 546-555, 2006

32. Santhiya D, Subramanian S and Natarajan K: Surface chemical studies on sphalerite and galena using extracellular polysaccharides isolated from Bacillus polymyxa. J Colloid Interface Sci 256: 237-248, 2002

33. Duh PD: Rebuttal on comparison of protective effects between cultured Cordyceps militaris and natural Cordyceps sinensis against oxidative damage. J Agric Food Chem 55: 7215-7216, 2007. 